\title{
Prevalence of alternative diagnoses in patients with suspected uncomplicated renal colic undergoing computed tomography: a prospective study
}

\author{
Julie Pernet, $\mathrm{MD}^{*}{ }^{\S}$; Sandra Abergel, MD*; Jerome Parra, MD ; Amine Ayed, $\mathrm{MD}^{\dagger}$; \\ Jerome Bokobza, $\mathrm{MD}^{*}$; Raphaelle Renard-Penna, $\mathrm{MD}^{\dagger}$; Isabelle Tostivint, $\mathrm{MD}^{\ddagger}$; \\ Marc-Olivier Bitker, MD, $\mathrm{PhD}^{\uparrow \S}$; Bruno Riou, MD, $\mathrm{PhD}^{* \S}$; Yonathan Freund, $\mathrm{MD}^{* \S}$
}

\section{ABSTRACT}

Objectives: Unenhanced computed tomography (CT) has become a standard imaging technique for uncomplicated renal colic in many countries. The appropriate timing of CT imaging has not been established, and guidelines recommend that this imaging be performed between 1 and 7 days of presentation. The primary objective of this study was to determine the prevalence of alternative diagnosis identified with low-dose unenhanced CT in the emergency department (ED) in patients with suspected uncomplicated renal colic.

Methods: This prospective single-centre study was carried out in a large university hospital ED. Over a 6-month period, all patients with clinically diagnosed renal colic and a plan to be discharged underwent low-dose unenhanced CT in the ED. Pregnant women, women of childbearing age not willing to have a pregnancy test, and patients who had already undergone diagnostic imaging were excluded. The primary outcome was the number and nature of the alternative diagnosis. Univariate analyses were performed to assess factors associated with the primary outcome.

Results: A total of 178 patients were screened, and 155 underwent $\mathrm{CT}$ in the ED. The mean age was 42.2 years; $69 \%$ were male. The diagnosis of uncomplicated renal colic was confirmed in 118 participants (76\%); 27 (17\%) had an inconclusive CT scan. Overall, 10 patients $(6 \%$; $95 \%$ confidence interval $[\mathrm{Cl}] 3-10$ ) had an alternative diagnosis, 5 of whom were subsequently hospitalized.

Conclusion: Low-dose unenhanced CT in the ED detects alternative diagnoses in $6 \%(95 \% \mathrm{Cl} 3-10)$ of patients with suspected uncomplicated renal colic, half of whom are subsequently hospitalized. Our prospective findings, which were similar to those reported in retrospective studies, are a potential argument for a systematic approach to ED imaging in suspected renal colic. Future research involving intervention and control groups would be helpful.

\section{RÉSUMÉ}

Objectif: La tomodensitométrie (TDM) sans densification est devenue, dans de nombreux pays, une technique d'imagerie courante dans les cas de colique néphrétique sans complications. Toutefois, le moment propice pour faire passer cet examen n'est pas établi, et, d'après les lignes directrices, l'examen devrait être effectué entre le premier et le septième jour de la consultation. L'étude avait pour objectif principal de déterminer la prévalence d'autres diagnostics que celui de colique néphrétique sans complications, posés dans des cas présumés, à l'aide de la TDM sans densification à faible dose, au service des urgences (SU).

Méthode: II s'agit d'une étude prospective, unicentrique, qui a été menée dans un SU d'un important centre hospitalier universitaire. Sur une période de 6 mois, tous les patients chez qui un diagnostic clinique de colique néphrétique avait été posé et pour lesquels la sortie était prévue ont passé une TDM sans densification à faible dose au SU. Les femmes enceintes, celles en âge de procréer qui ne souhaitaient pas passer un test de grossesse et les patients qui avaient déjà subi des examens d'imagerie diagnostique ont été écartés. Le principal critère d'évaluation était le nombre et la nature des autres diagnostics posés. Des analyses unidimensionnelles

From the *Emergency Department, Groupe Hospitalier Pitié-Salpêtriere, Assistance Publique - Hopitaux de Paris (APHP), Paris, France; †Radiology Department, Groupe Hospitalier Pitié-Salpêtriere, APHP, Paris, France; $¥$ Nephrology Department, Groupe Hospitalier Pitié-Salpêtriere, APHP, Paris, France; ๆUrology Department, Groupe Hospitalier Pitié-Salpêtriere, APHP, Paris, France; §Université Pierre et Marie Curie Paris 6 (Paris Sorbonne University), Paris, France.

Presented to the 2013 French Society of Emergency Medicine Annual Assembly, June 2013, Paris, France, and the 2013 American College of Emergency Physician Research Forum, October 2013, Seattle, WA.

Correspondence to: Dr. Yonathan Freund, Hôpital Pitie-Salpetriere - Service d'Accueil des Urgences, 47-83 Boulevard de I'Hôpital, Paris 75013, France.

This article has been peer reviewed. 
ont permis d'évaluer des facteurs associés au principal critère d'évaluation.

Résultats: Au total, 178 patients ont été sélectionnés, et 155 ont subi une TDM au SU. L'âge moyen était de 42.2 ans, et $69 \%$ des malades étaient des hommes. Le diagnostic de colique néphrétique sans complications a été confirmé chez 118 participants $(76 \%)$, tandis que I'examen s'est révélé non concluant chez 27 (17\%) patients. Dans l'ensemble, d'autres diagnostics ont été posés chez 10 patients $(6 \%$; intervalle de confiance à $95 \%$ [IC] 3-10), dont 5 ont dû être hospitalisés plus tard.

Conclusions: La TDM sans densification à faible dose, effectuée au SU conduit à la pose d'autres diagnostics que

Renal colic is a painful condition that accounts for some 1 million emergency department (ED) visits annually and has an incidence exceeding 1 per 1,000 persons per year in the United States. ${ }^{1,2}$ The evaluation of suspected renal colic with intravenous pyelography (IVP, also known as intravenous urography or IVU) has been replaced in many countries by other imaging modalities, in particular unenhanced computed tomography (CT) or the combination of ultrasonography and plain kidneyureter-bladder (KUB) radiography. Unenhanced CT has been found to have better diagnostic performance than IVP, with sensitivity and specificity rates over $95 \% .^{3-8}$ These diagnostic performances are also significantly better than a diagnostic approach combining KUB radiography and ultrasonography, particularly for stones less than $5 \mathrm{~mm}$ in size. ${ }^{6,7}$ For these reasons, the American College of Radiology recommends unenhanced CT as the technique of choice for renal stone imaging, ${ }^{9}$ as do the American Urological Association ${ }^{10}$ and the European Association of Urology. ${ }^{11}$ In France, ultrasonography is considered an acceptable first-line imaging modality according to both the French Society of Urology and the French Society of Emergency Medicine (AFU and SFMU). ${ }^{12}$ Frequently, imaging guidelines do not include a time frame recommendation. ${ }^{11,12}$ Some have proposed a time frame of imaging within 7 days for noncomplicated renal colic, ${ }^{13,14}$ and one study suggested that up to 3 weeks is acceptable. ${ }^{15}$

Although the use of CT in suspected renal colic has increased in recent years, ${ }^{2}$ less than half of such patients undergo this imaging in the ED., ${ }^{1,2}$ We sought to determine the merit of immediate CT in the ED in patients with suspected uncomplicated renal colic by determining the prevalence of alternative diagnosis identified with low-dose unenhanced CT in the ED in this population. celui de colique néphrétique sans complications dans $6 \%$ (IC à $95 \% 3-10)$ des cas présumés, et la moitié des patients concernés doit être hospitalisée plus tard. Les résultats de cette étude prospective, comparables à ceux d'études rétrospectives, offrent un argument possible en faveur de la réalisation systématique d'examens par imagerie au SU dans les cas présumés de colique néphrétique. II serait toutefois utile de mener d'autres études comportant cette fois des groupes témoins et des groupes expérimentaux.

Keywords: computed tomography, emergency department, renal colic

Low-dose CT has a radiation exposure that is slightly higher than that of KUB radiography and significantly less than that of IVP or regular-dose CT. ${ }^{16}$ Its diagnostic performance has been found to be similar to that of regular unenhanced CT. ${ }^{17}$

\section{METHODS}

This single-centre prospective study took place in the ED of our 2,000-bed urban university hospital, a department with a census of 52,000 emergency visits per year. We included patients from a 6-month period commencing on January 1, 2012. All patients who presented with abdominal or flank pain and were clinically diagnosed with uncomplicated renal colic before any imaging were screened for inclusion, and those who were eliglble subsequently underwent low-dose unenhanced CT scanning in the ED.

\section{Objectives}

The primary objective of this study was to determine the prevalence of diagnoses other than renal colic revealed by CT (alternative diagnosis) in patients with suspected uncomplicated renal colic. An alternative diagnosis was defined as a finding other than "noncomplicated renal colic" on the CT scan deemed to be related to the ED visit (e.g., renal colic complicated by fornix rupture or appendicitis). Incidental findings on the CT scan deemed to be unrelated to the ED visit were not categorized as alternative diagnoses. Our secondary objective was to determine whether variables existed that were significantly associated with the presence of an alternative diagnosis. 


\section{Inclusion and exclusion criteria}

The inclusion criterion was suspected uncomplicated renal colic. This diagnosis was made by the emergency physician after a clinical examination was performed and laboratory results were obtained. In our department, once complications have been clinically excluded for suspected renal colic, patients are routinely discharged with arrangements for urologic consultation and outpatient imaging within the next 7 days.

The initial investigation strategy for these patients, prior to radiologic imaging, included physical examination and blood tests for the purposes of excluding complications of urinary tract calculi. All patients with a clinical diagnosis of uncomplicated renal colic during the study period were then recruited in the trial and underwent low-dose unenhanced CT. Patients with potential complications of renal colic were excluded and underwent regular CT with or without contrast as indicated. Exclusion criteria were as follows:

- Urinary tract infection as defined by leukocytes or nitrites on urinary dipstick and confirmation by microbiologic direct examination

- Temperature $>37.5^{\circ} \mathrm{C}\left(99.5^{\circ} \mathrm{F}\right)$

- Absence of pain relief after treatment

- Known urinary tract structural abnormality, including a solitary or transplant kidney

- Suspicion of bilateral stones

- Impaired renal function (defined as an estimated glomerular filtration rate less than $80 \mathrm{~mL} / \mathrm{min}$ )

Any patients with diagnostic uncertainty of one or more of the above criteria were excluded as they were to be typically managed with an immediate CT in the ED. Prisoners, patients under 18 years of age, pregnant women, and women of childbearing age unwilling to undergo a pregnancy test were also excluded. The local Institutional Review Board approved the study and waived written consent (Comité de Protection des Personne - Paris Ile-de-France 6).

\section{Data collection}

All patients presenting to our ED during the study period with a clinical diagnosis of uncomplicated renal colic after emergency physician assessment were prospectively screened, and data were collected regarding their demographics, vital signs, pathology results, treatment, and outcome. If the diagnosis of uncomplicated renal colic was confirmed by the emergency physician at discharge and no radiologic studies had been performed, then the patient was included in the trial and a low-dose unenhanced abdominal CT scan was obtained.

\section{Radiologic examination}

We used a 128-slice helical CT scanner with 90 mAs/ slice, $1.5 \mathrm{~mm}$ width, and $0.75 \mathrm{~mm}$ increments (Philips Healthcare, Cleveland, OH). CT images were interpreted by a senior radiologist and corroborated by a second senior radiologist. Two senior emergency physicians blindly reviewed each participant's complete medical file (including clinical observations in the ED, laboratory results, urine microscopy, and CT results) and classified patients into two groups: "uncomplicated renal colic" and "alternative diagnosis." If there was disagreement in the categorization of a case, a third physician was consulted.

\section{Statistical analysis}

Variables are presented as mean (with standard deviation), when normally distributed, and median (with interquartile range), when skewed according to the Kolgomorov-Smirnov test. Proportions were compared with the Fisher exact method, means were compared with the Student $t$-test, and medians were compared with the Mann-Whitney $U$ test. All statistical tests were two-tailed, and a $p$ value of less than 0.05 was deemed to be statistically significant. Interrater agreement was calculated using the kappa statistic.

\section{Sample size}

Previous retrospective studies suggest that the proportion of suspected renal colic patients with alternative diagnoses is just over $10 \% .^{18,19} \mathrm{We}$ hypothesized that the prevalence of alternative diagnoses would be higher than $5 \%$. Assuming a prevalence point estimate of $10 \%$ for alternative diagnoses, we calculated that a sample size of 140 participants would be necessary to obtain a $95 \%$ confidence interval (CI) with a lower limit greater than $5 \%$.

As approximately 300 patients per year are discharged from our ED with the diagnosis of uncomplicated renal colic, we decided to enrol patients for a 6-month period with a target of including 150 patients. 


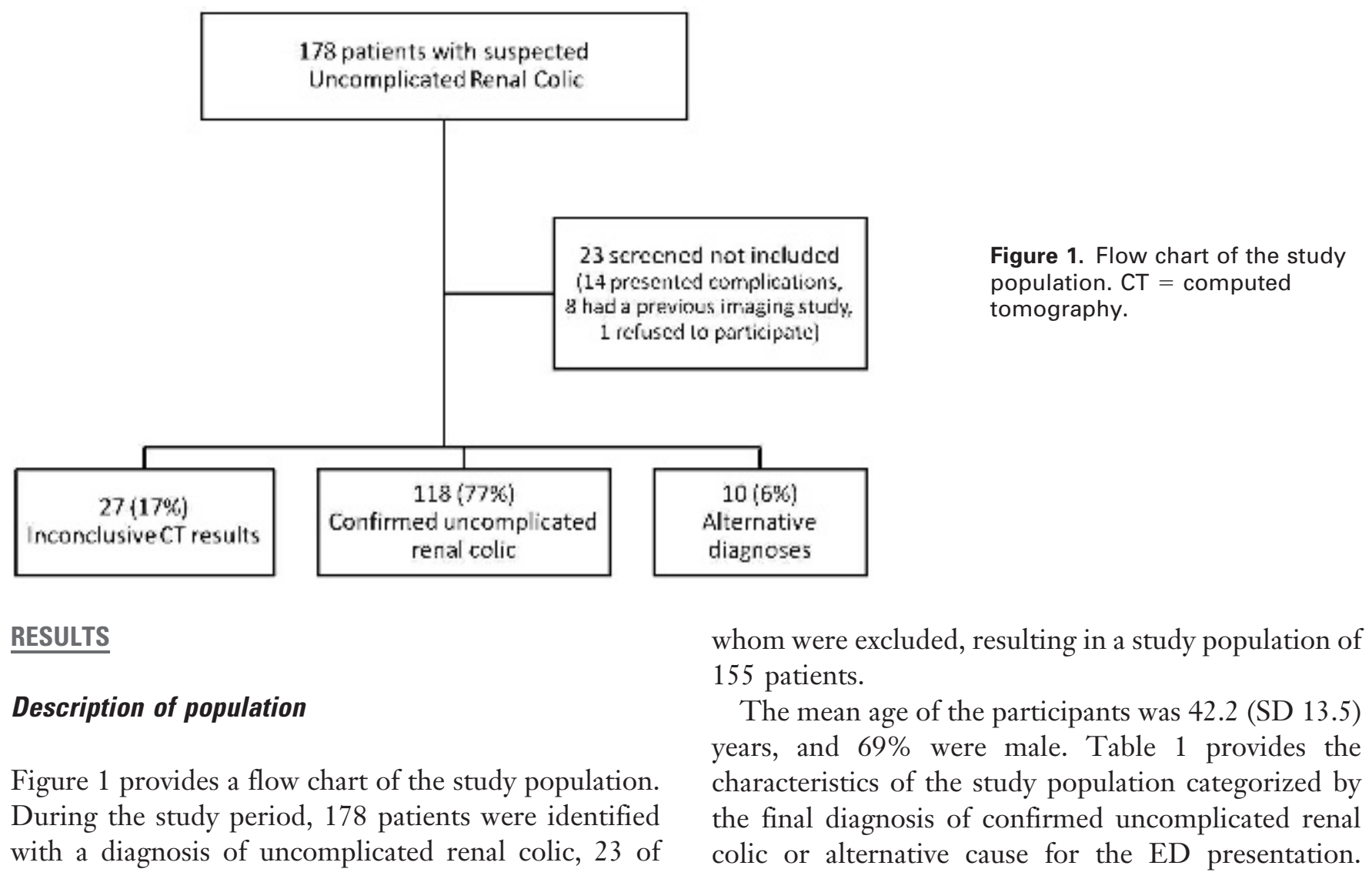

\begin{tabular}{|c|c|c|c|}
\hline Characteristic & Total & Confirmed diagnosis & Alternative diagnosis \\
\hline$n(\%)$ & 155 & $145(94)$ & $10(6)$ \\
\hline Male sex & $107(69 \%)$ & $70 \%$ & $50 \%$ \\
\hline Age, mean (SD) & $42.2(13.5)$ & $41.8(13.5)$ & 46.5 (13.9) \\
\hline Analogue pain scale on admission, median (IQR) & $3(0-6)$ & $3(0-7)$ & $5(2-7)$ \\
\hline Pulse, median (IQR) & 75 (67-89) & $76(65-86)$ & $80(69-90)$ \\
\hline History of renal colic & $68(44 \%)$ & $44 \%$ & $40 \%$ \\
\hline Temperature $\left({ }^{\circ} \mathrm{C}\right)$, mean $(\mathrm{SD})$ & $36.5(0.5)$ & $36.5(0.5)$ & $36.7(0.6)$ \\
\hline Creatinine $(\mu \mathrm{mol} / \mathrm{L})$, mean (SD) & $79.3(18.6)$ & $80.0(18.5)$ & $69.3(14.0)$ \\
\hline Leukocytes (G/L), mean (SD) & $9.5(3.3)$ & $9.6(3.2)$ & $8.9(4.2)$ \\
\hline \multicolumn{4}{|l|}{ Urinary dipstick } \\
\hline Leukocytes $(N=149)$ & $22(14 \%)$ & $13 \%$ & $30 \%$ \\
\hline Erythrocytes & $123(79 \%)$ & $83 \%$ & $83 \%$ \\
\hline Nitrites & $3(2 \%)$ & $2 \%$ & $0 \%$ \\
\hline \multicolumn{4}{|l|}{ Analgesics administered } \\
\hline Acetaminophen $(N=155)$ & $72(46 \%)$ & $46 \%$ & $42 \%$ \\
\hline NSAID & $98(63 \%)$ & $65 \%$ & $67 \%$ \\
\hline Tramadol/codeine & $40(25 \%)$ & $25 \%$ & $34 \%$ \\
\hline Morphine & $71(46 \%)$ & $49 \%$ & $50 \%$ \\
\hline Stone detected on $\mathrm{CT}$ & $121(78 \%)$ & & \\
\hline Unexpected differential diagnosis & $10(6 \%)$ & & \\
\hline Hospitalization & $5(3 \%)$ & & \\
\hline
\end{tabular}


Hematuria was present in $79 \%$ of cases, and most of the study population received morphine in the ED. A calculus was detected in 121 (78\%) patients, and an alternative diagnosis was identified in 10 cases $(6 \%$; $95 \%$ CI 3-10). The kappa for interrater agreement between the two emergency physicians reviewing the charts was 0.90 (95\% CI 0.77-0.99), with a raw agreement of $99 \%$. The alternative diagnoses identified were two cases of large calculi needing urologic intervention, two cases of epiploic appendagitis, and one case each of renal infection, biliary colic, appendicitis, ileitis, partial small bowel obstruction, and intrarenal hemorrhage. Five patients (3\% of total; $95 \%$ CI 1-7) who would have been discharged were admitted; three underwent a double-J stent placement within the next 24 hours, one underwent appendicectomy, and one was admitted for a tumour of the colon and subsequently underwent surgery.

\section{Uncomplicated renal colic}

A diagnosis of confirmed uncomplicated renal colic was made in 118 patients, of whom 73 (62\%) had hydronephrosis. The median number of stones visualized on a CT scan was 1 (interquartile range 1,1 ). Calculi were detected on the right side in 53 patients (44\%), the left side in 48 patients (42\%), and bilaterally in 14 patients (12\%). The most common calculus location was the ureter (95 patients, $81 \%$ ).

In the univariate analysis, a lower creatinine level was the only factor significantly associated with the identification of another diagnosis after unenhanced CT: 69.3 (SD 14) $\mu \mathrm{mol} / \mathrm{L}$ versus 80.0 (SD 18) $\mu \mathrm{mol} / \mathrm{L}$, $p=0.04$.

\section{DISCUSSION}

In recent years, most guidelines and studies have endorsed CT as the imaging modality of choice for renal colic. ${ }^{10,11}$ Accordingly, the use of CT in the evaluation of ED patients with flank pain has significantly increased. Westphalen and colleagues reported a 10-fold increase in the use of CT between 1996 and 2007. ${ }^{1}$ Whether or not it is important to perform CT at the time of the ED encounter remains unclear. Recent studies have found that the use of CT for evaluating suspected renal colic in the ED remains low, with less than half of patients undergoing CT as a first-line imaging study. ${ }^{1}$ In a recent French study,
$49 \%$ of physicians indicated that they perform unenhanced CT as a first-line technique in the evaluation of patients with suspected renal colic. ${ }^{20}$

In our study, we found that among patients with suspected uncomplicated renal colic who would have been discharged home from the ED, 6\% (95\% CI 310) had an alternative diagnosis, half of whom were subsequently hospitalized. To our knowledge, this is the first study that has prospectively evaluated the benefit of unenhanced CT in the ED for such patients. Previous studies have retrospectively determined the proportion of cases with incidental findings on the CT scan. Nadeem and colleagues reported that unenhanced CT yielded an incidental diagnosis rate of $18 \%$ in studies ordered by emergency physicians. ${ }^{21}$ Three retrospective studies reported that the proportion of cases with an alternative diagnosis ranged between 8 and $12 \% .{ }^{19,22,23}$

The reluctance of physicians to systematically obtain CT scans for suspected uncomplicated renal colic is likely, at least in part, to be due to efforts to limit radiation exposure, particularly in young women. However, CT for renal colic in ED patients should be promoted for several reasons. First, in our prospective study, the rate of alternative diagnosis was relatively high at $6 \%$ and included patients with surgical pathology, roughly half of whom were subsequently hospitalized. Second, some form of imaging is mandatory for suspected renal colic, with KUB radiography/ultrasonography being an acceptable alternative to unenhanced CT in some guidelines. ${ }^{12}$ The radiation exposure of low-dose unenhanced CT is only slightly higher than KUB radiography and significantly lower than regular-dose unenhanced CT (1-1.9 v. $0.5-1$ v. $4.5-5 \mathrm{mSv}$, respectively)..$^{16,24,25}$ Lowdose unenhanced CT appears to have emerged as the imaging modality of choice given its very low radiation, diagnostic performance similar to that of regular-dose unenhanced CT, and significantly better diagnostic performance than KUB/ultrasonography. ${ }^{17}$ Finally, although the population with the greatest concerns regarding radiation exposure is women, this also appears to be the population with the highest rate of alternative diagnosis. In our sample, $10 \%$ of women had an alternative diagnosis on low-dose unenhanced CT compared to $5 \%$ of men. Although this difference was not statistically significant, it is consistent with previous reports: Nadeem and colleagues reported a higher rate of incidental findings in women, ${ }^{21}$ and 
Chowdhury and colleagues found a twofold decrease in the positive rate for urolithiasis on CT in women. ${ }^{26}$

Katz and colleagues raised the problem of reattendance in patients with renal colic, reporting that $4 \%$ of patients in their study underwent three or more CT scans in 6 years. ${ }^{27}$ However, their study involved normal-dose CT, and the authors estimated that these $4 \%$ of patients received at least $10 \mathrm{mSv}$ in the 6 years of the study period. Although low-dose unenhanced CT radiation exposure is approximately fivefold lower than regular-dose unenhanced $\mathrm{CT}$, the total radiation dose in patients with multiple visits could reach a substantial amount. As CT is recommended for the evaluation of renal colic, our study focused on the timing of the scan. Total radiation exposure in patients with a history of renal colic who are likely to be re-evaluated with lowdose unenhanced CT merits further investigation.

Our study has limitations that should be considered. First, we calculated that our sample size was an estimated event rate that was higher than the $6 \%$ we found, and as a result, the lower boundary of our $95 \%$ CI was less than expected. Second, the small number of events in our sample may have been the reason we identified only one factor associated with an alternative diagnosis on univariate analysis and may have prevented us from confirming previous reports suggesting that other factors are associated, such as sex and the presence of urine leukocytes. Third, we had a relatively high number of inconclusive CT scans. Twenty-seven (17\%) CT scans were reported as showing no pathologic findings, a result attributable to either the patient having already passed a stone, not having renal colic in the first place, or a missed calculus (false negative scan). These inconclusive studies increased our denominator and thus lowered the prevalence of alternative diagnoses, although none were confirmed to represent uncomplicated renal colic. Conversely, the fact that all patients included in the study subsequently received imaging could have lowered the threshold for physicians to conclude, without imaging studies, that uncomplicated renal colic existed. This could have resulted in an enrolment bias and increased the number of patients included in the study, hence potentially increasing the prevalence of alternative diagnoses that we found. Fourth, "absence of adequate pain relief after treatment" was an exclusion criterion. With neither a strict definition of "pain relief" nor timing for its assessment, this criterion was left to the physician in charge, potentially biasing our findings.
Finally, our main limitation was that we did not compare our protocol to usual care, including outpatient CT. To prove that systematic unenhanced CT improves the management of suspected renal colic in the ED, a study that compares the two management strategies in terms of prognosis, reattendance, and radiation exposure would be required. Validation of our findings would require an interventional study with a control group.

\section{CONCLUSION}

Low-dose unenhanced CT in the ED detects alternative diagnoses in $6 \%(95 \%$ CI 3-10) of patients with suspected uncomplicated renal colic, half of whom are subsequently hospitalized. Our prospective findings, which were similar to those reported in retrospective studies, are a potential argument for a systematic approach to ED imaging in suspected renal colic. Future research involving intervention and control groups would be helpful.

Competing interests: None declared.

\section{REFERENCES}

1. Westphalen AC, Hsia RY, Maselli JH, et al. Radiological imaging of patients with suspected urinary tract stones: national trends, diagnoses, and predictors. Acad Emerg Med 2011;18:699-707, doi:10.1111/j.1553-2712.2011.01103.x.

2. Brown J. Diagnostic and treatment patterns for renal colic in US emergency departments. Int Urol Nephrol 2006;38:87-92, doi:10.1007/s11255-005-3622-6.

3. Miller OF, Rineer SK, Reichard SR, et al. Prospective comparison of unenhanced spiral computed tomography and intravenous urogram in the evaluation of acute flank pain. Urology 1998;52:982-7, doi:10.1016/S0090-4295(98)00368-9.

4. Wang J-H, Shen S-H, Huang S-S, Chang C-Y. Prospective comparison of unenhanced spiral computed tomography and intravenous urography in the evaluation of acute renal colic. 7 Chin Med Assoc 2008;71:30-6, doi:10.1016/S1726-4901(08) 70069-8.

5. Sourtzis S, Thibeau JF, Damry $\mathrm{N}$, et al. Radiologic investigation of renal colic: unenhanced helical CT compared with excretory urography. AfR Am $\mathcal{7}$ Roentgenol 1999; 172:1491-4, doi:10.2214/ajr.172.6.10350278.

6. Worster A, Preyra I, Weaver B, Haines T. The accuracy of noncontrast helical computed tomography versus intravenous pyelography in the diagnosis of suspected acute urolithiasis: a meta-analysis. Ann Emerg Med 2002;40:2806, doi:10.1067/mem.2002.126170.

7. Varma G, Nair N, Salim A, Marickar YMF. Investigations for recognizing urinary stone. Urol Res 2009;37:349-52, doi: $10.1007 / \mathrm{s} 00240-009-0219-\mathrm{z}$. 
8. Middleton WD, Dodds WJ, Lawson TL, Foley WD. Renal calculi: sensitivity for detection with US. Radiology 1988;167: 239-44.

9. Coursey CA, Casalino DD, Remer EM, et al. ACR Appropriateness Criteria ${ }^{\circledR}$ acute onset flank pain-suspicion of stone disease. Ultrasound Q 2012;28:227-33, doi:10.1097/ RUQ.0b013e3182625974.

10. Fulgham PF, Assimos DG, Pearle MS, Preminger GM. Clinical effectiveness protocols for imaging in the management of ureteral calculous disease: AUA Technology Assessment. 7 Urol 2012 Oct 18. [Epub ahead of print].

11. Türk C, Knoll T, Petrik A, et al. Urolithiasis. Available at: http://www.uroweb.org/gls/pdf/18_Urolithiasis.pdf (accessed February 2013).

12. El Khebir M, Fougeras O, Le Gall C, et al. [2008 update of the 8th Consensus Development Conference of the Francophone Society of Medical Emergencies of 1999. The treatment of adult renal colic by the emergency services and in emergency rooms]. Prog Urol 2009;19:462-73, doi:10.1016/j.purol.2009. 03.005 .

13. Bultitude M, Rees J. Management of renal colic. BMF 2012; 345:e5499, doi:10.1136/bmj.e5499.

14. Wright PJ. Managing acute renal colic across the primarysecondary care interface: a pathway of care based on evidence and consensus. BMF 2002;325:1408-12, doi:10.1136/bmj. $\underline{325.7377 .1408}$.

15. Lindqvist K, Hellström M, Holmberg G, et al. Immediate versus deferred radiological investigation after acute renal colic: a prospective randomized study. Scand 7 Urol Nephrol 2006;40:119-24, doi:10.1080/00365590600688203.

16. Kluner C, Hein PA, Gralla O, et al. Does ultra-low-dose CT with a radiation dose equivalent to that of KUB suffice to detect renal and ureteral calculi? 7 Comput Assist Tomogr 2006;30:44-50, doi:10.1097/01.rct.0000191685.58838.ef.

17. Niemann T, Kollmann T, Bongartz G. Diagnostic performance of low-dose CT for the detection of urolithiasis: a meta-analysis. AfR Am $\mathcal{F}$ Roentgenol 2008;191:396-401, doi: 10.2214/AJR.07.3414.

18. Cullen IM, Cafferty F, Oon SF, et al. Evaluation of suspected renal colic with noncontrast CT in the emergency department: a single institution study. 7 Endourol 2008;22: 2441-5, doi:10.1089/end.2008.0120.

19. Patatas K, Panditaratne N, Wah TM, et al. Emergency department imaging protocol for suspected acute renal colic: re-evaluating our service. Br $\mathcal{F}$ Radiol 2012;85:1118-22, doi: $10.1259 / \mathrm{bjr} / 62994625$.

20. Meria P, Bensalah K, Bringer J-P, et al. Résultats d'une enquête de pratiques auprès des urologues français concernant la prise en charge des calculs et de la lithiase urinaire en 2012. Prog Urol FMC 2012;22:F56-62, doi:10.1016/j. fpurol.2012.04.003.

21. Nadeem M, Ather MH, Jamshaid A, et al. Rationale use of unenhanced multi-detector CT (CT KUB) in evaluation of suspected renal colic. Int 7 Surg 2012 Nov 13. [Epub ahead of print].

22. Jo H, Buckley BT. Assessment of referral patterns for CT KUB in a tertiary setting. 7 Med Imaging Radiat Oncol 2009; 53:516-21, doi:10.1111/j.1754-9485.2009.02117.x.

23. Ahmad NA, Ather MH, Rees J. Incidental diagnosis of diseases on un-enhanced helical computed tomography performed for ureteric colic. BMC Urol 2003;3:2, doi: 10.1186/1471-2490-3-2.

24. Van Der Molen AJ, Cowan NC, Mueller-Lisse UG, et al. CT urography: definition, indications and techniques. A guideline for clinical practice. Eur Radiol 2008;18:4-17, doi:10.1007/s00330-007-0792-x.

25. Caoili EM, Cohan RH, Korobkin M, et al. Urinary tract abnormalities: initial experience with multi-detector row CT urography. Radiology 2002;222:353-60, doi:10.1148/radiol. 2222010667.

26. Chowdhury FU, Kotwal S, Raghunathan G, et al. Unenhanced multidetector CT (CT KUB) in the initial imaging of suspected acute renal colic: evaluating a new service. Clin Radiol 2007;62:970-7, doi:10.1016/j.crad.2007.04.016.

27. Katz SI, Saluja S, Brink JA, Forman HP. Radiation dose associated with unenhanced CT for suspected renal colic: impact of repetitive studies. A7R Am $\mathcal{7}$ Roentgenol 2006;186: 1120-4, doi:10.2214/AJR.04.1838. 